\title{
Laboreal
}

Volume $15 \mathrm{~N}^{\circ} 1$ | 2019

Trabalho e cooperação

\section{Os incentivos à produção, seus efeitos na segurança e na saúde do trabalho e entre coletivos de trabalho: uma abordagem da formação em ergonomia}

Los incentivos a la producción, sus efectos en la seguridad y salud laboral y entre los colectivos de trabajo: una aproximación desde la formación en ergonomía Les incitations à la production, ses effets sur la sécurité et santé du travail et les collectifs de travail : une approche de la formation en ergonomie The incentives to the production, its effects in the safety and work health and among the work collectives: an approach from training in ergonomics

\section{Pamela Astudillo y Carlos Ibarra}

\section{OpenEdition}

Journals

Edición electrónica

URL: http://journals.openedition.org/laboreal/1393

DOI: 10.4000/laboreal. 1393

ISSN: 1646-5237

Editor

Universidade do Porto

\section{Referencia electrónica}

Pamela Astudillo y Carlos Ibarra, « Os incentivos à produção, seus efeitos na segurança e na saúde do trabalho e entre coletivos de trabalho: uma abordagem da formação em ergonomia », Laboreal [En línea], Volume 15 N$^{0} 1$ | 2019, Publicado el 01 julio 2019, consultado el 11 abril 2020. URL : http:// journals.openedition.org/laboreal/1393; DOI : https://doi.org/10.4000/laboreal.1393

Este documento fue generado automáticamente el 11 abril 2020.

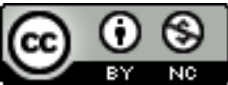

Laboreal está licenciado com uma Licença Creative Commons - Atribuição-NãoComercial 4.0 Internacional. 


\section{Os incentivos à produção, seus efeitos na segurança e na saúde do trabalho e entre coletivos de trabalho: uma abordagem da formação em ergonomia}

Los incentivos a la producción, sus efectos en la seguridad y salud laboral y entre los colectivos de trabajo: una aproximación desde la formación en ergonomía Les incitations à la production, ses effets sur la sécurité et santé du travail et les collectifs de travail : une approche de la formation en ergonomie The incentives to the production, its effects in the safety and work health and among the work collectives: an approach from training in ergonomics

Pamela Astudillo y Carlos Ibarra

Manuscrito recibido en: Diciembre/2018

Aceptado tras peritaje: Mayo/2019

Agradecemos a: Ana María Seifert, Conseillère en santé et sécurité chez Confédération des syndicats nationaux de Quebec, Nicole Vezina, académica de l'Université du Québec à Montréal, Canadá y a Julia Medel, socióloga del Centro de Estudios de la Mujer de Chile, por su colaboración y participación en los cursos de formación en ergonomía y género para dirigentes sindicales e inspectores de SST.

\section{Introducción}

\subsection{Contexto de Trabajo y Empleo en Chile}

1 La Fuerza de Trabajo Ocupada en Chile es de aproximadamente 8.500.000 trabajadores, sólo un $70 \%$ de ellos se encuentra dentro del empleo formal, con contrato de trabajo y 
cobertura de protección social, habiendo una tasa de participación de $71 \%$ para los hombres y un $45 \%$ para las mujeres (Instituto Nacional de Estadísticas, 2018). Existe, además, una estructura del empleo formal marcada por la división sexual del trabajo, en sentido vertical y horizontal en la mayor parte de la industria, notablemente a causa de la influencia del taylorismo (Astudillo \& Ibarra, 2014). Chile, al igual que otros países en desarrollo, intentó adecuarse a las nuevas realidades de la globalización, desregulando su economía y flexibilizando su aparato estatal, todo ello con el fin de capturar recursos multinacionales lo que le permitió incrementar su tasa de inversión y creación de empleo. El empleo formal sufrió una sistemática precarización a partir de los años 80s, en plena dictadura militar, con la implantación de un modelo económico neoliberal centrado en la exportación de materias primas y el debilitamiento de los sectores industriales (Manky, 2018; Valdés, Rebolledo, Pavez \& Hernández, 2014; Ffrench-Davis, 2014) propiciando la flexibilización laboral en un contexto de derechos laborales acotados (Manky, 2018). Por contraparte, proliferaron los sectores de servicios, comercio y el sector financiero, así como una fuerte privatización de las empresas estatales sumado a los cambios en los regímenes de trabajo, pasando de la mano de obra directa a la subcontratación en todos los sectores de actividad económica (Valdés et al., 2014; Ffrench-Davis,2014). Es así como en la actualidad más de la mitad de las empresas del territorio nacional mantiene alguna de sus funciones en régimen de subcontratación, incluyendo la actividad principal, siendo posible encontrar trabajadores subcontratados en áreas tan diversas como la construcción, la agricultura, la minería, los servicios de salud y otros servicios sociales, el transporte, el comercio, e incluso en la mayoría de las instituciones públicas.

2 Por otra parte, en el mismo periodo de los años 80's y por medio del denominado "Plan Laboral", se realizaron importantes reformas estructurales, en función de la "libertad económica" y la abertura económica internacional, con efectos negativos para los trabajadores, con la reducción del nivel y cobertura del salario mínimo, se facilitó el despido de los trabajadores y se eliminaron los juzgados del trabajo (siendo restablecidos en 1986), se limitaron las atribuciones de los sindicatos impidiendo la negociación colectiva inter-empresa y por ramas, restringiendo los derechos de los dirigentes sindicales y promoviendo así la segmentación y atomización sindical (Ffrench-Davis, 2014), limitando su capacidad de defensa de sus representados, lo que debilitó la acción colectiva, la asociatividad y la cooperación entre los trabajadores.

3 La incorporación de la flexibilidad laboral condujo a denominar las nuevas formas de trabajo como "atípicas" y/o "excepcionales", caracterizadas por horarios de trabajo atípicos, jornadas especiales extendidas en sistemas de turnos, así como la figura de " enganchadores" de mano de obra y "contratistas", que dieron lugar a distintas formas de externalizar funciones en las empresas y en el Estado, cambiando profundamente la relación entre capital y trabajo (Valdés et al, 2014). Uno de los efectos más conocidos de estas reformas neoliberales es la "dualización" que es la división entre una minoría de trabajadores que tienen empleos estables, un salario regular y condiciones de trabajo decentes y aquellos que pasan a la cadena de subcontratación con pérdida de ese estatus (Kalleberg, 2011). Si bien la dualización es una tendencia global, pocos países han experimentado una transformación como Chile, donde por ejemplo los trabajadores mineros subcontratados pasaron de representar el $12 \%$ de la fuerza laboral a más del 60\% entre 1990 y 2000 principalmente, en labores de servicio (Manky, 2018). 
4 En los años 90's los cambios incorporados a la legislación laboral normalizaron y legitimaron la matriz de la flexibilidad laboral (Ffrench-Davis, 2014) incluida la subcontratación. Además en los años posteriores, se afianzaron los mecanismos de remuneración basados en el trabajo a destajo (trabajo a trato en Chile o pagado a la pieza en otras partes del mundo) y la incorporación de mecanismos de incentivos salariales colectivos o individuales para alcanzar metas de producción más elevadas, representando estos últimos la principal forma de acceder a mejoras salariales y cuya práctica también se da en los empleos con salarios regulares, tanto en las instituciones y empresas públicas como privadas, siempre con el fin de "mejorar la productividad y la eficiencia".

\subsection{La participación en Salud y Seguridad en el Trabajo en Chile}

A pesar de los profundos cambios en el modelo económico y en las relaciones laborales en Chile, el marco jurídico que da la Ley 16.744 del Ministerio del Trabajo y Previsión Social a la Seguridad Social en Salud y Seguridad en el Trabajo (SST), no ha presentado modificaciones sustantivas desde su promulgación en 1968. La Ley resta importancia al rol sindical en la prevención en SST, solo se hace mención a sus funciones descritas en el Código del Trabajo que los faculta para plantear observaciones a los Comités Paritarios de Higiene y Seguridad, que tienen representación de empleadores y trabajadores, pero cuya validez social es cuestionada por parte de los trabajadores, dada la asimetría de poder decisional con el empleador, lo que quedó de manifiesto durante el proceso de elaboración de la Política Nacional de SST. Esta situación de asimetría en la representación para la toma de decisiones, ha sido ampliamente descrita en el contexto europeo y norteamericano (Boix \& Vogel, 2013), además las tasas de sindicalización y de negociación colectiva, son mucho más bajas en América Latina, particularmente en Chile que está muy por debajo del promedio de los países que integran la Organización para la Cooperación y el Desarrollo Económico (OCDE).

6 En otro sentido, algunos avances se han presentado en el último periodo a raíz de la discusión impulsada por el accidente de los 33 mineros de la Mina San José el año 2010, lo que llevó a Chile a ratificar el año 2011 el Convenio 187 de la Oficina Internacional del Trabajo (OIT) y adoptar una Política Nacional de SST en un marco tripartito el año 2016. Esto ha permitido un mayor dinamismo en las regulaciones de SST, donde se ha estipulado la necesidad de la Ergonomía como disciplina para estudiar y mejorar las condiciones de trabajo, principalmente de los problemas músculo-esqueléticos y psicosociales, requiriendo de formación especializada y de una masa crítica que evalúe los contextos de trabajo. Es así como, la formación se ha concentrado en postítulos profesionales y postgrados, sin mayor vinculación con la formación de trabajadores en este ámbito, en contraste con los países de América del Norte y de Europa, donde la ergonomía se ha posicionado como una herramienta para apoyar las demandas de los trabajadores(as) por mejores condiciones de trabajo a través de sus sindicatos o agrupaciones. El conocimiento y las soluciones frente a los problemas de salud laboral se encuentran, hoy en día, fuertemente dominado por un enfoque "técnico", característico de expertos e incluso científicos, en administración y gestión, vigorosamente influenciado por las normas de calidad, lo que con frecuencia desvaloriza la validez e importancia del conocimiento y experiencia de los propios 
trabajadores (Boix \& Vogel, 2013; Guérin, Laville, Daniellou, Duraffourg, \& Kerguelen, 2008).

\subsection{La Cooperación y la Organización del Trabajo}

7 La cooperación es el trabajo realizado por un grupo de personas que poseen objetivos similares y descansa sobre la división de las tareas, a diferencia de la coordinación que consiste en enunciar la orden de trabajar conjuntamente. Según Dejours (2009), la posibilidad de construir la cooperación en el trabajo depende del cómo está organizado el trabajo. Es común que en algunos trabajos su organización afecte a las relaciones interpersonales y promueva el individualismo, la competencia, lo cual desarma el tejido de apoyo mutuo y la solidaridad entre colegas de trabajo.

Por otra parte, son innegables las consecuencias positivas que traen para la salud de los trabajadores y trabajadoras las relaciones interpersonales positivas, entre ellas la cooperación. Existe evidencia relacionada con las condiciones físicas y psicológicas del trabajo a destajo en el sector manufacturero (Premji, Lippel \& Messing, 2008), donde las relaciones interpersonales se ven frecuentemente deterioradas como respuesta del sistema de trabajo pagado por incentivos individuales. Este es un ámbito de la interacción social que ha sido estudiada ampliamente desde distintos puntos de vista (Herranz, 2015) y desde hace un tiempo que en las áreas de recursos humanos y en la administración de los trabajos se han comenzado a utilizar prácticas de gestión que han generado deterioro de la cooperación, reforzando la competitividad, principalmente orientando a los trabajadores hacia la obtención de metas de producción inalcanzables.

La ergonomía puede representar una estrategia para implicar a las personas en la planificación y control de una parte significativa de su trabajo, con el suficiente conocimiento y poder para influir sobre los procesos y sus resultados, con el objetivo de conseguir metas deseables para los trabajadores y las empresas.

\subsection{La Formación en Salud y Seguridad en el Trabajo y Ergonomía}

Una concepción clásica de formación en SST, desde la aproximación de los factores humanos, consiste en la instrucción y las prácticas destinadas a adquirir habilidades y conocimiento relativos a las reglas, conceptos o actitudes necesarias para trabajar en un contexto de tarea específica, abordando el reconocimiento de los riesgos y sus medidas de control, el aprendizaje de prácticas de trabajo seguras, el uso del equipo de protección personal, la adquisición de conocimientos sobre medidas de emergencia y acciones preventivas (Cohen \& Colligan, 1998; Montreuil \& Lancomblez, 2013). Habitualmente representa prescripciones sobre el cómo trabajar y el entrenamiento en dichas prácticas. Sin embargo, la formación en el ámbito del trabajo no se debe limitar a las simples demostraciones de gestos profesionales y transmisión de instrucciones, en lo que se denomina el trabajo prescrito (Guérin et al., 2008).

11 La formación en SST para los trabajadores, desde la aproximación de la ergonomía de la actividad, es contextualizada por la situación de trabajo que ellos vivencian (St-Vincent et al., 2011). De hecho, el análisis de la actividad se ha instalado y estructurado como una aproximación productiva en las ciencias de la educación y la formación (Yvon \& Durand, 2012; Baril-Gingras, Bellemare \& Brun, 2007), siendo la formación-acción un 
recurso para la acción y una fuente de información para la investigación (Yvon \& Durand, 2012).

La formación-acción en ergonomía de la actividad, en general, reconoce la experiencia y el conocimiento de los participantes, se centra en aprender haciendo, y en utilizar el conocimiento operativo de los participantes sobre el funcionamiento de la organización (Baril-Gingras et al., 2007) y en las estrategias que ellos han desarrollado para afrontar la situación de trabajo en un contexto dado (Lacomblez, ollagnier \& Teiger, 2016), así como para conducir a transformaciones en los contextos de trabajo (Baril-Gingras et al., 2007).

Más allá de la transferencia clásica de conocimiento asociada con la capacitación, la mejora de varios aspectos de una situación de trabajo no se puede lograr sin la contribución de la experiencia y el conocimiento de los que allí trabajan (Montreuil \& Lancomblez, 2013; Denis et al., 2013). Es así como se busca cambiar las representaciones individuales en los actores, aprovechando el intercambio de su saber-hacer en la situación colectiva de formación (Brunet, Viel, Presselin \& See, 2005) en un proceso de co-aprendizaje o de formación recíproca (Teiger et al., 2014) a fin de potenciar la toma de decisiones, sistematizando información para introducir o apoyar la mejora de situaciones laborales (Messing, Lefrançois \& Saint-Charles, 2018; Montreuil \& Lancomblez, 2013).

Considerando lo descrito, se realizó una serie de formaciones en ergonomía para dirigentes sindicales e inspectores de SST, que buscaba identificar los riesgos para la salud relacionados con la actividad de trabajo y sus determinantes, la comprensión del impacto de la variabilidad de las personas y de los contextos de trabajo, así como aquello que favorezca la eficacia de las intervenciones, incorporando también un enfoque de género. Con una modalidad de formación-acción, fue posible comprender la importancia de este tipo de formación, lo que permitió generar un documento de apoyo para la formación en ergonomía y género para dirigentes sindicales (Astudillo, Ibarra \& Medel, 2015).

El objetivo de esta publicación es mostrar que a través de la formación-acción en ergonomía, es posible aportar conocimientos prácticos a los dirigentes sindicales y a los inspectores de SST, a través del modelo del análisis de la actividad centrado en la persona (Vézina, 2001; St-Vincent et al., 2011), por medio del cual ellos reconocen los determinantes de la actividad de trabajo y se acercan a posibles pistas de solución. Un objetivo secundario, es presentar un análisis de los casos revisados en las formaciones, donde las modalidades de organización del trabajo, el ambiente de trabajo, el tipo de remuneración, los incentivos, los horarios atípicos, etc., impactan en las representaciones de SST y en las dinámicas cooperación que se establecen entre colectivos de trabajo y cómo este conocimiento contribuyó para retroalimentar las futuras formaciones, representando un aporte a la comprensión de los efectos que conllevan en las personas algunas prácticas laborales.

\section{Metodología}

16 Esta publicación se basa en el análisis de 9 formaciones cortas (de 4 días) en ergonomía y género, a partir de las verbalizaciones grabadas, a un total de 76 participantes (52 dirigentes sindicales y 24 profesionales de SST). Los casos estudiados mediante el análisis de la actividad de trabajo (Guérin, et al., 2008) fueron en diversos puestos de 
trabajo y rubros productivos de Chile. De los 76 casos de estudio, se eligieron 15, los cuales tenían vinculación con el trabajo a destajo con modalidad de pago de incentivos con bono de producción y donde se veían afectadas las relaciones humanas como la cooperación.

\subsection{La Formación:}

La formación fue realizada en pequeños grupos de 7 a 12 participantes, contó con 2 fases: La primera una Fase no presencial: Preparación/Recolección de datos y la segunda Fase presencial: Presentación de casos estudiados/ Análisis de la actividad. El objetivo fue que los participantes desarrollen habilidades de observación y fiscalización para avanzar en la evaluación del trabajo y de manera secundaria adoptar un rol activo en temas de SST en sus trabajos, pudiendo analizar los problemas bajo los principios de la ergonomía y el género.

En la Fase no presencial, el participante recibió la consigna de elegir una actividad de trabajo en su empresa para analizar, recabar antecedentes del puesto de trabajo y material audiovisual (fotos y/o videos), realizar verbalizaciones y entrevistas al trabajador(es), para lo cual se les entrega una pauta de preguntas abiertas que deben ser contestadas por el trabajador del puesto de trabajo. Estos datos deben ser entregados previamente a la realización de la fase presencial, lo cual se relaciona con un proceso de aprendizaje como un proceso interno de entendimiento, que se produce cuando el estudiante participa activamente en la comprensión y elaboración del conocimiento, aludiendo al constructivismo social.

\subsection{El Análisis de la Actividad de trabajo}

En la fase presencial guiada por dos ergónomos y un sociólogo, los participantes reciben el contenido del cuadro teórico de la ergonomía de la actividad, con la ayuda del modelo de la situación de trabajo centrada en la persona en actividad (Vézina, 2001), como se puede ver en la figura 1, a través del cual se analizan las situaciones de trabajo aportadas por cada participante. La situación de trabajo corresponde a un estado que puede describirse en sus diversos elementos y que es experimentado por personas situadas en un entorno de trabajo particular, es decir, una configuración particular de determinantes. Las relaciones entre las personas y el lugar de trabajo son parte de la situación de trabajo (St-Vincent et al., 2011). 
Figura 1 - Modelo de la situación de trabajo centrada en la persona en actividad de Vézina, 2001 sacado y traducido de St-Vicent et al 2011

CONDICIONES Y MEDIOS OFERECIDOS POR EL LUGAR DE TRABAJO Organización - del trabajo - de la producción - de la formación Ambiente físico Dispositivos técnicos FISICA Y MENTAL

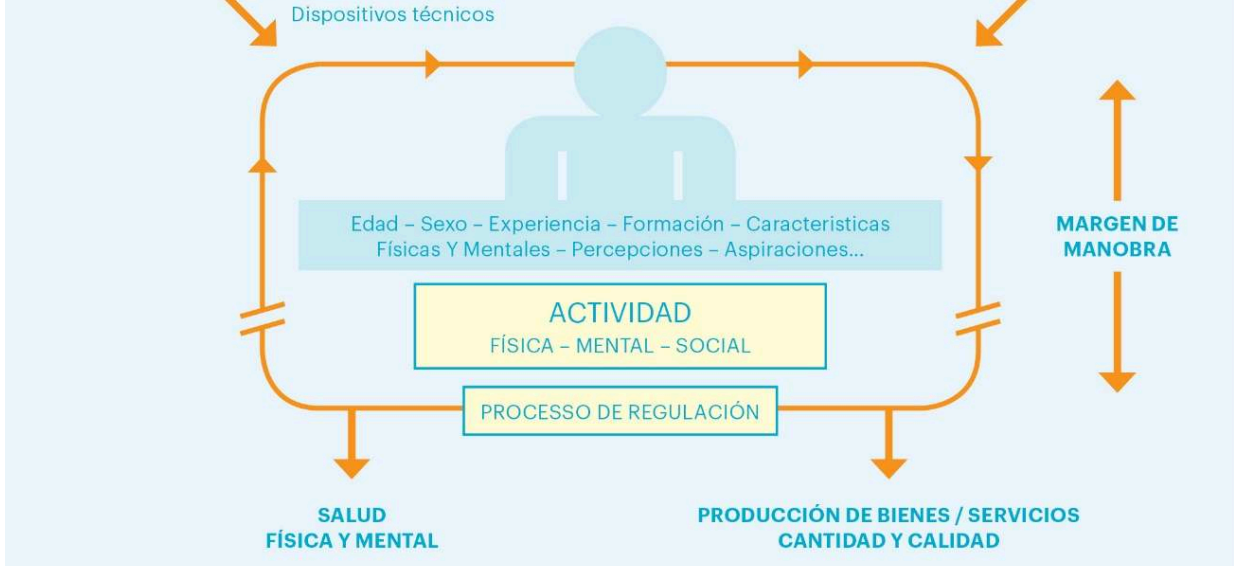

AMBIENTE SOCIAL Estructuras sociales y cultura Relaciones funcionales y jerárquicas Relaciones con los clientes y usuarios

TAREAS Y EXIGENCIAS Procedimientos y consignas

Figura 1: Modelo de la situación de trabajo centrada en la persona en actividad de Vézina, 2001, sacado y traducido de St-Vincent et al. 2011. entendidos como un elemento de la situación laboral que está en el origen de la forma en la cual la persona podrá realizar su actividad (St-Vincent et al., 2011), tienen que ver con las condiciones que influencian en el actuar del trabajador, dentro de los cuales podemos encontrar los incentivos que otorga la administración al trabajador(a) para aumentar la producción de bienes y servicios, pero, por otra parte, al determinar el cómo de la actividad también podrá manifestar efectos en la salud física y mental de la persona. La identificación de los determinantes es obviamente un paso muy importante en la intervención ergonómica, ya que permitirá modificar los elementos de la situación laboral.

Los participantes aplican estos conocimientos analizando los casos que han aportado de manera individual. Cada participante presenta su caso, entrega los antecedentes y la información recolectada en el proceso de preparación. En esta fase, se identifican las operaciones, los modos operatorios, los factores de riesgo asociados, se reflexiona sobre el impacto de la variabilidad de las características individuales de las personas en situación de trabajo, en particular el género. Se identifican los determinantes de la actividad y los puntos de apoyo que podrían favorecer las intervenciones en el medio del trabajo. Esto propicia un aprendizaje colaborativo, bajo el paradigma del constructivismo social, en el cual se construye el aprendizaje a partir de la información entregada y las experiencias del colectivo de participantes (Cenich \& Santos, 2005).

\subsection{Formación-acción en ergonomía}

Este tipo de formación-acción permite a los dirigentes sindicales enriquecer sus demandas, apelar sus problemáticas no reconocidas o invisibilizadas y contribuye a 
orientar adecuadamente a sus representados y para los inspectores de SST, enriquecer sus prescripciones en el control de las condiciones de trabajo (Ibarra \& Astudillo, 2014). Para ambos aporta conocimientos sobre ergonomía (Teiger et al., 2014) para argumentar mejor los cambios que se requieren en los lugares de trabajo, lo cual se refuerza al conocer el contexto chileno de Salud Ocupacional y los recursos que tienen los trabajadores donde recurrir.

En general, en ambos grupos se generan alianzas entre los participantes (dirigentes sindicales o inspectores) y comparten estrategias de cómo actuar bajo su rol, respondiendo a los mandatos que ambos tienen en temas de SST. El rol técnico-político en el caso de los fiscalizadores y el rol político-técnico en el caso de los dirigentes en SST.

\subsection{Balance al finalizar la jornada de formación}

Al finalizar cada jornada se realizó un balance para identificar aquellos determinantes que más se repetían entre las situaciones analizadas, con especial atención en aquellos macro-determinantes que están vinculados a la organización del trabajo.

\section{Resultados}

Se constató que un macro determinante de la actividad, en muchos de los contextos de trabajo analizados, fue el trabajo a destajo, particularmente en la industria agroalimentaria del Salmón, la Fruticultura, la Pesca y Cultivos de productos del mar, pero también en el Transporte, Textiles y Servicios de Salud, cuyo importante impacto sobre la SST se refleja en el elevado número de Trastornos Musculo-esqueléticos (TME) $\mathrm{y}$ en el debilitamiento de las relaciones interpersonales entre colegas, supervisores, etc., producto de la falta de cohesión y la competencia que se genera por el sistema de trabajo, lo cual no era claro en inicio de la formación para los participantes. Además, el análisis de la actividad en contexto de formación permitió generar cambios en la representación del problema, ampliando las razones por las cuales los trabajadores están presentando efectos para su salud, propiciando una reflexión crítica sobre la importancia de una visión colectiva del trabajo, donde la cooperación entre colegas puede representar un factor de protección para afrontar los problemas en SST, al reconocer elementos que facilitan el progreso y aquellos que obstaculizan el mejoramiento de las condiciones laborales, así como la necesidad de construir más instancias de participación y aprendizaje.

A continuación, se presentan los casos analizados, seleccionados para esta publicación, los cuales se describirán los efectos para la salud, el deterioro de las relaciones interpersonales que están sujetos a las exigencias de producción y las condiciones de trabajo pagado por incentivo de producción:

\subsection{Casos: Industria agroalimentaria del Salmón}

El inspector de SST que aportó este caso, revisó las condiciones de SST de una empresa de procesamiento de salmones en las líneas de producción más importantes, destacando la situación laboral de la trabajadora que realizaba el corte de cabeza de salmón, al inicio de la línea de producción. La trabajadora verbalizó dificultades en la 
actividad que realiza, tales como: el espacio restringido y cuchillos defectuosos. Ella había logrado acceder a un puesto de trabajo masculinizado, donde la remuneración era más elevada en comparación con otras líneas del proceso donde había solo mujeres. $\mathrm{Su}$ actividad de trabajo se caracteriza por ciclos breves y repetitivos de 4 segundos, siendo este factor de riesgo uno de los más evidentes. Sin embargo, ni para el inspector que asistió a la formación, ni para la trabajadora que participó en el estudio era evidente la necesidad de discutir sobre el sueldo variable y el bono de producción asignado por la empresa, representando este el determinante de la actividad más importante, dado que el incentivo es el principal factor que intensifica el trabajo osteomuscular de las personas en la empresa, lo que solo se evidenció en la descripción detallada de la actividad de trabajo.

Un trabajo similar fue aportado por una dirigenta sindical de una empresa de salmones, al comparar la situación de su empresa respecto de aquella comentada anteriormente, se puso en evidencia que a pesar de las deplorables condiciones de trabajo, que no tenían relación con el género, en comparación con su empresa, existía la posibilidad de que las mujeres tuvieran acceso a los puestos "masculinizados" que son mejor remunerados, lo que le había sido negado en su empresa. Esto particularmente es evidente cuando señala: “...como dirigenta yo estoy sorprendida de ver una mujer en ese puesto de trabajo, al que muchas veces hemos solicitado a la gerencia de incluir mujeres y se nos ha negado, ahora con este ejemplo podemos exigir a la empresa que incorpore mujeres en este puesto..." Además, la dirigenta expresó al finalizar el curso su apreciación sobre cómo los conocimientos adquiridos, que le permitirán tener mayores herramientas para fundamentar las mejoras al trabajo de sus representados, diciendo: “...como dirigente yo integro el comité paritario y muchas veces en el análisis causal de las enfermedades usaba el experto mucho lenguaje y yo no sabía cómo defenderme ante eso...necesitaba algo así que me abriera los ojos que me permitiera demostrar cómo un trabajador se me accidento... ahora tengo bases suficientes para hablar con el ergónomo..., Nosotros vimos que la única forma de que el comité paritario funcione es que haya un dirigente sindical....".

Un tercer caso del mismo rubro fue aportado por un inspector. La actividad de trabajo analizada fue el despinado del salmón, que también es una actividad repetitiva de alta frecuencia en los movimientos de los dedos de las trabajadoras (puesto feminizado), con un elevado número de TME. El ergónomo de la aseguradora prescribió pausas para ejercicios compensatorios y el desarrollo de una herramienta, diseñada tomando en cuenta la antropometría de las trabajadoras. No obstante, al igual que en los casos anteriores, la remuneración incluía importantes incentivos por producción, aunque en este caso el incentivo era colectivo, por puesto de trabajo y no individual como en los casos anteriores. Esto en lugar de disminuir la intensificación del trabajo, la mantenía disminuyendo la competitividad entre las trabajadoras, pero limitando las posibilidades de rotación para cambiar los patrones de exposición. El mismo inspector que aportó este caso posteriormente expresó la importancia de considerar a la persona en actividad, subrayando la utilidad del análisis de la actividad, mostrando que él comprendió la razón por la cual la incorporación de un dispositivo técnico no logró modificar la situación de trabajo, lo cual manifiesta en la siguiente frase: ... "Ahora, puedo incorporarlo a mi práctica pidiéndole a la compañía que realice un estudio antes de hacer cualquier cambio. Además, antes estaba orientado al riesgo y ahora comprendo que debo centrarme también en la persona". 
30 Un cuarto caso analizado de esta industria fue el trabajo de eviscerado del salmón, el cual se realiza aguas arriba en la línea con respecto a los puestos anteriores. Aquí la trabajadora, estando de pie toda la jornada, debía tomar con su mano el salmón, con una máquina succionar las vísceras y depositar el salmón eviscerado en la línea, en un ciclo de trabajo que dura 3 segundos y se repite continuamente. Este trabajo, aportado por una inspectora de SST, también presentaba un número elevado de TME y era remunerado por bono de producción individual, por lo cual una vez más quedaban en entredicho las medidas preventivas, como las pausas para ejercicios y la rotación, que por los mecanismos de incentivo estaban impedidas.

\subsection{Casos: Fruticultura}

31 Las inspectoras de SST aportaron 3 casos para analizar de este rubro, en todos ellos el motivo de su elección tenía que ver con los riesgos de TME. El primero se trata de una empresa productora de peras de exportación a Europa y América del Norte. En la línea de producción existía un número importante de TME. Pero, en su mayoría eran rechazados en la calificación como patología laboral por la aseguradora de SST. Al analizar la actividad de trabajo del puesto de embalaje, fue posible develar que las trabajadoras deben manipular las peras, envolverlas en papel y luego depositarlas en cajas, embalar las cajas y comenzar de nuevo otro ciclo que se repite durante toda la jornada. Las trabajadoras intensificaban su actividad, por el elevado ritmo de la cadena productiva en el packing (embalaje), considerando que este trabajo se paga por "trato diario" más un incentivo por producción individual. Las trabajadoras se ven afectadas a causa de este macro determinante, con un estrecho margen de maniobra para la regulación, por cuanto no tienen descanso o pausa dentro de la jornada laboral, más allá de la colación. Por otra parte, para la inspectora de SST, se hacía complejo poder revertir esta situación en la empresa, dado que, al no haber reconocimiento de la patología laboral, la empresa no genera mayor cambio. Al cierre de la formación la inspectora refirió lo siguiente: “...Hay aseguradoras que hacen sus evaluaciones de las estaciones de trabajo sin la persona y después rechazan las enfermedades de los trabajadores. Sabíamos que era malo, pero no teníamos un argumento sólido para hacerles hacer su trabajo de manera diferente...". Esto también refleja la mayor comprensión de la situación de trabajo y de la profundidad de las evaluaciones, al mismo tiempo que les da mayor fundamento al momento de exigir cambios en la empresa.

32 En un segundo caso, proveniente de otra región de Chile, las trabajadoras debían seleccionar manzanas de exportación y descartar aquellas de calidad regular, para mantenerlas en el mercado nacional. En este trabajo las personas (principalmente mujeres) también verbalizaron dolencias en sus muñecas, manos, brazos, hombros, etc. Si bien, los ciclos de trabajo eran unos segundos más holgados, el mecanismo de incentivos impedía la rotación de puestos al estar asociadas a la posibilidad de alcanzar mayor salario para la persona. Este puesto lleva a la persona a "especializarse" en una determinada tarea sin dejar margen a la persona para pasar por otras tareas que demanden diversas competencias, situación que no era objeto de una reflexión, pese a la existencia del sindicato en la empresa.

33 El tercer caso analizado fue el trabajo de mantenimiento de las parras (arboles) de uva de mesa de exportación, realizado por un trabajador, que debía cortar los sarmientos (ramas), lo cual generaba una exposición importante a factores de riesgos de TME. La 
verbalización del trabajador, refiriendo dolencias a nivel de los hombros, cuello y columna lumbar y la observación del video daba cuenta de ambos brazos sobre el nivel de los hombros la mayor parte del tiempo, al sostener la herramienta mientras hacía el corte. Sin embargo, el determinante de la actividad que se relaciona con la organización del trabajo y la producción era el pago a trato por jornada mediante incentivo individual, el hecho de que el trabajador era remunerado por cada árbol que cortaba y sobre aquello un incentivo por volumen producido en la jornada no parecía algo evidente ni para la inspectora ni para el trabajador. En este contexto, la ausencia de sindicato y la poca cohesión del colectivo de trabajadores reforzaba la idea de que cada trabajador podía mejorar su trato negociando individualmente con el empleador aumento del salario, pero en desmedro de la SST.

\subsection{Casos: Pesca y Cultivos de productos del mar}

34 El dirigente sindical, buzo mariscador profesional experto con 18 años de experiencia, solicita a la administración de su empresa que realicen cambios en el sistema de trabajo de los buzos, que deben incorporarse al mar con equipos que se suman al peso de la extracción de boyas y redes con pescados y cultivos de mariscos, desde la profundidad del mar establecida habitualmente a los 20 metros, lo que ha generado efectos en la salud de los trabajadores, como TME, problemas de estrés y cardiovasculares.

Se analiza la necesidad y se observa que esta actividad además de los determinantes asociados a los dispositivos técnicos (equipos y herramientas), las condiciones de trabajo en hiperbaria y las condiciones propias de trabajar bajo el agua, también está determinada por los incentivos salariales, en concreto es pagado por día en que bucea, compuesto por el salario mínimo y se añaden bonos de eficiencia, a fin de evitar el ausentismo a lo que él se refería así: “...A nosotros nos pagan por día trabajado, si yo buceo me gano mi sueldo, si no lo hago no gano nada, esas son las condiciones, no nos pagan bonos productivos, eso lo gana la gente en tierra en la planta, nosotros tenemos bonos de eficiencia, donde tenemos que ganarlo si no faltamos al trabajo ningún día del mes...". Esto denotaba que los trabajadores acceden a un mayor salario (sobre el mínimo legal) si no se ausentan del trabajo, no obstante, eso también estimulaba el trabajar aun cuando las condiciones de salud no eran las adecuadas.

Otro aspecto que llama la atención en este caso, es que en la misma empresa, es posible mantener un sindicato que representa al colectivo de trabajadores de los cultivos de productos del mar, principalmente integrado por hombres. Y por el contrario, en la planta de procesamiento de los productos, donde las líneas de producción son feminizadas, hay ausencia de sindicato, debido a las dificultades de este colectivo para mantenerlo, siendo despedidos sus integrantes ante el menor interés en organizarse. Se presenta la paradoja de que existe posibilidad de asociatividad y cooperación entre los trabajadores para formular sus demandas en un sector de la empresa, mientras que, en la otra parte, se fomenta una cultura de competitividad a través de la intensificación de la producción por medio de los incentivos o bonos de producción individuales. Cabe destacar que, en la planta de procesamiento referida, existía un elevado número de TME y remuneración por incentivos individuales con diversas escalas de bonificación donde, para acceder a la primera escala de bono, las trabajadoras deben hacer 36 kilogramos de productos del mar limpios por día. Si no lo logran, quedan con el salario mínimo, eso les obliga a trabajar a un ritmo intensificado. Las actividades de 
procesamiento de productos del mar, se ven menos valoradas lo cual se traduce en un bono de producción más débil, las trabajadoras hacen un trabajo muy exigente y minucioso, debiendo respetar elevados criterios de calidad.

Por otra parte, el trabajador reafirma su compromiso con el colectivo de trabajo, y la necesidad de apoyo mutuo en beneficio de la salud de todos: “...Yo apoyo mucho a la empresa, para que la empresa tire para arriba, pero no le creo cuando va en desmedro de un trabajador, ese es mi trabajo para eso los trabajadores me eligieron sobre todo cuando se trata de la salud..." Representa la vaga sensación de seguridad que tiene el trabajador y al mismo tiempo la expresión de solidaridad por otros trabajadores y refleja la poca esperanza en el sistema de SST, expresando lo siguiente: “... lamentablemente hay que decirlo hay muchos buzos a lo largo de la costa, yo trabajo en una empresa que de alguna forma me respalda, que de alguna forma me cuida un poquito, me protege entre comillas, pero hay tantos buzos que no tienen empresa, ¿Quién protege a mis compañeros? ¿Dónde está el sistema?”.

En respuesta a la opinión anterior, otro dirigente del sector transporte comenta las dificultades expuestas por el buzo, luego de haber realizado el análisis de la actividad, lo lleva a comprender las exigencias y la precariedad de un rubro ajeno al suyo, manifestando lo siguiente: “...Un trabajo no tiene que dar pena y me angustio mucho ver que hay trabajadores que sí sufren cada día que van a trabajar, me quebró no puedo aceptar eso, que un ser humano tenga que sufrir para ir a trabajar".

\subsection{Casos: Transporte}

Un dirigente sindical, conductor de trenes metropolitanos, solicita a la administración modificar los asientos de las cabinas del tren, por contar con dimensiones inapropiadas para los conductores y una mesa de control que no permite visualizar bien los indicadores críticos. Los conductores sufren lesiones asociadas al trabajo, a nivel de extremidad inferior, columna lumbar y como consecuencias de las interacciones con el público, tensiones psicológicas, trastornos ansiosos, del sueño, etc. Con el análisis de la actividad, se detalla el trabajo y se indican las posibilidades que tiene esta actividad de ser modificada a nivel de micro y macro determinantes. Sin embargo, una de las cosas que más sorprende es el hecho de que los propios trabajadores del servicio de transporte también son evaluados en función de su productividad, se generan incentivos salariales para evitar el ausentismo y de esa manera, mantener la operación constante del servicio, sin que ello implique una reflexión de hasta dónde estos esquemas de remuneración han penetrado todos los sectores de actividad productiva y de servicios.

Otro dirigente aportó su caso como Maquinista de trenes de carga provenientes de la minería. Esta actividad consiste en conducir un tren de carga, lo cual se hace de día y de noche, con jornadas promedio de 11 horas y un máximo de 12 horas, se conduce un tren con una o dos locomotoras. Siendo particular la exigencia cuando se conduce con una sola locomotora ya que la mitad de la jornada se hace manejando al revés en trayectos cortos, dado que las locomotoras tienen solo un frente de manejo y no se giran para cambiar el sentido de marcha, por lo cual gran parte del tiempo el trabajador está sentado con la columna torcida y el cuello girado. El dirigente se refería a su horario de trabajo de la siguiente manera: “...Nuestro trabajo se mide por horas mensuales y es de 180 horas, no tenemos máximo semanal ni máximo diario, en nuestra empresa hemos conseguido un máximo de 12 horas por jornada, pero en otras trabajan hasta 16 horas diarias...". A la 
pregunta ¿considera usted que la cantidad de trabajo es excesiva? relataba: “...Totalmente y categóricamente encuentro excesivo, nuestras autoridades, todos se han pronunciado al respecto, pero aún no legislan para solucionar este tema que lleva muchos años..."

Otro dirigente del sector transporte de autobuses urbanos, refería que las malas condiciones de trabajo, expresadas en la falta de mantenimiento de las máquinas, las vibraciones, exceso de calor en el verano y la violencia de los pasajeros, generan en los conductores permanentes dolencias osteomusculares en espalda y cuello. Además, se sentían estresados por la extensa jornada y la fuerte presión de mantener la operación el sistema en los horarios punta. Para esto, la empresa "premiaba" con bonos la "ausencia de faltas" durante el mes.

42 Es importante considerar que en estos tres casos se daba la presencia de sindicatos "fuertes" con capacidad de negociación colectiva, donde este tipo de bonos también era objeto de interés en la negociación por parte de los trabajadores y empresa.

43 Al finalizar el curso, el dirigente ferroviario expresa la necesidad de mayor cohesión de los trabajadores organizados ante el contexto adverso que se percibe para la SST, reflejando un sentido de solidaridad y cooperación con las organizaciones pequeñas, planteando lo siguiente: “...Allí tenemos que trabajar, los sindicatos poderosos que tienen mayor capacidad, mayor organización deben trabajar para que organizaciones más pequeñas tengan la posibilidad de defenderse de mejor manera..."

\subsection{Casos: Sector Textil}

Este rubro fue uno de los más afectados por las reformas económicas de los años 80's, lo cual con la introducción de productos principalmente manufacturados por las maquiladoras de Asia, India, México, etc. pasó prácticamente a desaparecer en nuestro país. A pesar de aquello, algunas actividades aún se conservan, pero ya no en la fábrica como solía ser. Es la propia dirigenta sindical que participa de estas formaciones, quien devela la realidad de trabajo y refiere que esta actividad ha sido desplazada a las casas de los trabajadores-as, donde hoy se ensamblan camisas, blue jeans, pantalones y otras vestimentas, que llegan desde el exterior y se entregan en una larga cadena de subcontratación a las grandes empresas comercializadoras. Las trabajadoras están expuestas a TME y a otros riesgos, teniendo en cuenta que el pago es por pieza (cuellos, cierres o mangas de camisas), estando totalmente al margen de la seguridad social.

Los determinantes de este trabajo son la carencia de condiciones físicas en el ambiente de trabajo, realizado dentro de sus propias casas, con máquinas de su propiedad. La dirigenta textil lo representa de la siguiente manera: “...laborar en el hogar no debiera ser impedimento para sentirnos con los mismos derechos que cualquier otro trabajador... Nosotras necesitamos que nuestro trabajo se visibilice..."

Además, se toman en el análisis otros 3 casos que fueron aportados por inspectores de SST, particularmente las actividades textiles estaban orientadas a surtir de ropa de trabajo a las empresas mineras en el norte de Chile, acá la actividad si bien no se realizaba en los hogares de los trabajadores y trabajadoras, si se desarrollaba en pequeños talleres de 4 a 8 máquinas máximo. En todos los casos, el salario se estructuraba a partir del sueldo mínimo, para luego aumentar con bonificación por prenda de vestir terminada. 


\subsection{Casos: Servicios de Atención de Salud}

Un dirigente paramédico, en su petitorio, demandaba a la administración del hospital comprar una silla con apoya brazos adecuados y un mesón a la altura correcta. Se evidenció la necesidad de mejorar la iluminación, el mobiliario adecuado para la actividad del laboratorio, la necesidad de controlar la exposición a los agentes químicos, entre otros. En este caso, pese a que la trabajadora tenía una sola petición (la silla y la luz), al final del análisis de la actividad, se pudo develar que hay otros elementos que se pueden solicitar para mejorar las condiciones actuales de trabajo. En este caso, los incentivos de producción estaban vinculados a cumplir con los tiempos de respuesta los clientes para tener los resultados de los exámenes.

Otro caso aportado por las dirigentas sindicales de los servicios de salud tienen que ver con la atención de pacientes en el servicio dental de atención primaria de salud (APS). En este caso, la trabajadora odontóloga, con 3 años de experiencia en su puesto, tenía como principal queja la sobrecarga de trabajo y la falta de personal. En su actividad de atención de pacientes para la limpieza y el tratamiento de caries, demandaba repetición de movimientos en posturas forzadas en muñecas, hombros, espalda y cuello en flexión y rotación. El trabajo requiere de gran concentración y atención sensorial al evaluar el estado del paciente. Ella refería como principal dificultad el tener que responder a las metas de calidad y producción del servicio dado que, además de su salario fijo, recibía el servicio completo un bono por cumplimiento de una meta sanitaria, asociada al número de atención de pacientes.

Esta realidad se repite en prácticamente todas las actividades de atención de pacientes en el ámbito público, donde los bajos salarios y las altas demandas de atención se compensan a través del pago de bonos asociados a programas de mejoramiento de gestión (PMG), que son transversales en toda la administración pública, cuyas metas no son objeto de una negociación colectiva, dado que en el sistema público no está consagrado este derecho, por lo tanto, son muchas veces impuestas por quienes organizan el trabajo a distancia desde los niveles centrales de los servicios.

Al finalizar el curso, una trabajadora apela a la necesidad que tienen los dirigentes sindicales de profundizar los conocimientos en ergonomía y participar de las instancias decisionales que tiene la empresa, manifestando: “...Es importante que los dirigentes sindicales estudien, no conocen que hace el comité paritario, y eso hace que los prevencionistas hagan el informe de los accidentes y culpen de todo al trabajador, si los dirigentes no conocen que van a defender... además nosotros tenemos fuero..."

\section{Discusión}

51 La ventaja de establecer un puente entre los aspectos teóricos de una formación en ergonomía de la actividad, con la posibilidad de aplicar esos contenidos en un caso práctico, hace valorar el trabajo que desempeñan hombres y mujeres en los distintos sectores productivos analizados. Se develan las precariedades del empleo formal, como las modalidades de subcontratación y las estructuras salariales variables. Además, se visibilizan las brechas de género, en el acceso al empleo formal y la segregación dentro de este, en los cuales se encuentra mayoritariamente a las mujeres vinculadas a la categoría de trabajos feminizados que, al tener esa connotación, reciben menor atención a las condiciones de trabajo que se ofrecen para los trabajadores(as) y lo que 
se asocia con el supuesto de que "el trabajo femenino no tiene riesgos", por lo tanto, los riesgos al no ser estudiados, son invisibilizados (Messing, 2000; Astudillo \& Ibarra, 2014).

Así como en la problemática de género, inherente en los contextos de trabajo a causa de la división sexual del trabajo, prevalece una organización del trabajo basado en obsoletas prácticas de producción de bienes y servicios, donde el trabajo a destajo puede parecer arcaico (Premji et al., 2008) y que, por ejemplo, están en oposición a combatir los riesgos psicosociales. Desde este punto de vista, la relación que tienen los incentivos a la producción con el deterioro de la SST se vincula con los efectos en las relaciones interpersonales que esto provoca, observándose en la frustración experimentada por los trabajadores, por la intensificación de la actividad y no poder lograr una óptima performance, la relación entre los pares se debilita, hay falta de diálogo entre trabajadores y empleadores.

Las dificultades de asociación entre los colectivos de trabajo, alejan las posibilidades de hacer efectivos los derechos de los trabajadores, a causa de las asimetrías en la relación de poder (Boix \& Vogel, 2013). Esto impide el desarrollo sano de las sociedades y comunidades. En ergonomía, se plantea que esta tendencia es creciente. Por ejemplo, Loriol, Boussard, \& Caroly (2005) señalan que la policía francesa evalúa a los policías en términos de "números" de arrestos, etc., lo que dificulta la prevención de problemas de salud ocupacional. Esto se suma a lo observado en el relato de los maquinistas de trenes y autobuses, donde se les evalúa por la ausencia de inasistencias al trabajo o más bien por disponibilidad y, en el caso de la odontóloga, evaluados por el número de pacientes tratados.

Por otra parte, una explicación desde la sociología refiere que los sujetos se caracterizan porque practican asiduamente estrategias de cooperación para sobrevivir (Herranz, 2015), se ve mediada por la reacción de los trabajadores(as) cuando la organización del trabajo (Dejours, 2009) impulsa a aumentar la cantidad de trabajo, imponiendo una elevada frecuencia, altas exigencias, bajo control, bajo apoyo (Theorell, Karasek, \& Eneroth, 1990) y las escasas compensaciones en términos de seguridad en el empleo (Siegrist, 1996), intercambiando condiciones de trabajo por ganar más dinero. Frente a estas ofertas, la reacción hacia la colaboración ya no es la misma. Desde esta perspectiva importa mantener el empleo, la remuneración, obtener protección social y respeto de los mínimos derechos, aunque a veces esos mínimos derechos no sean respetados. Esto podría explicar que dentro de los colectivos de trabajo la cohesión es variable y los procesos de identificación grupal, están en relación con el nivel de compromiso que desarrollan las personas con el trabajo (Teiger et al., 2014).

El fomentar un juicio crítico con un desarrollo de la visión sistémica de la ergonomía aplicada al trabajo, tanto para los dirigentes sindicales, como para los inspectores, era poco evidente para los participantes de las formaciones en ergonomía y género. Durante la fase presencial, cada participante al explicar los detalles del trabajo estudiado a los demás participantes expresaba conocimientos del trabajo, la visión desde la persona, lo cual era reforzado y se les motivaba a continuar apropiándose de estos conocimientos. Para los ergónomos, la formación de actores en el conocimiento del trabajo no es más que un medio para lograr acciones sobre el trabajo (Teiger et al., 2013). Por otra parte, los inspectores destacaron la posibilidad de conocer una aproximación en ergonomía que era diferente de lo que comúnmente prevalece en la 
SST en el país, como es la aplicación de listas de chequeo, ya que dentro del modelo de la "situación de trabajo centrado en la persona en actividad" (Vézina, 2001) es un constructo para la explicación y el desarrollo de las intervenciones ergonómicas en las organizaciones y disminuir los efectos negativos para la persona y para la organización. Para ellos, representó "abrir los ojos" hacia otro tipo de conocimiento en ergonomía que estaba centrado en la actividad de la persona y su dimensión personal del trabajo, lo cual para ellos no era evidente ya que concebían esta disciplina desde una mirada de experto.

De manera secundaria, para los dirigentes sindicales, la formación en ergonomía significaba nivelar sus conocimientos en temas de SST, ejerciendo el rol de velar. Como también, referían su actuar partiendo de las estrategias de convencimiento y posicionamiento de los temas de SST. En el caso de los inspectores, lograron valorar a la persona en actividad, que sufre los efectos de las condiciones de trabajo ofrecidas por el empleador y que a veces son poco visibles para el personal de SST de las empresas. De igual modo, para ambos grupos de participantes, relevaron al trabajador, con sus percepciones, aspiraciones individuales en el trabajo, de conocimiento que tienen la variedad de contextos analizados. Tanto inspectores como los dirigentes compartieron experiencias y las estrategias en función de la inspección que pueden tener los dirigentes sindicales dentro de su rol y en el caso de los inspectores de SST. La formación propone aprendizajes que suponen nuevos puntos de vista de uno mismo, de otras personas, de las cosas, de saberes (Teiger et al., 2014). Por esto, cada vez es más frecuente que se utilice la formación para prevenir, por ejemplo, el riesgo músculoesquelético y para lo cual los ergónomos tienen el rol importante en concebir formaciones adecuadas a los trabajadores y trabajadoras para que desarrollen competencias y prevención.

57 Para ambos, la formación implicó conocer estrategias que les ayudan a manejar conflictos internos de sus organizaciones y aportan conocimientos a dirigentes con menos experiencia en el quehacer sindical, lo cual promueve a la acción y representa la movilización de todos los saberes para una sola causa (Teiger et al., 2014), nutriendo su propias prácticas y reflexiones. En el caso de los fiscalizadores, se observó un avance en la modificación de la representación de las expectativas de su rol como entidad de salud y de control en los procesos vinculados al trabajo. Sin embargo, el análisis del trabajo mostró que es necesario actuar de manera estratégica, generando cooperación con otros actores de SST, como los dirigentes sindicales, sobre todo durante las inspecciones a las empresas, donde acostumbran a actuar individualmente.

Por otra parte, los conocimientos que produce la ergonomía, adquieren especial relevancia para los dirigentes sindicales, ya que, en el proceso de intervención ergonómica, la participación de los actores internos de la empresa es fundamental, denominada "construcción participativa", debido a que en su desarrollo implica un proceso de interacción entre la ergonomía y los actores decisionales, trabajadores concernientes y diversas especialidades del establecimiento (St-Vincent et al., 2011). En este marco, la posibilidad que tienen los dirigentes sindicales, de colaborar en un proceso de intervención movilización (Brunet et al., 2005; Ibarra \& Astudillo, 2014) no es menor, ya que ellos identifican problemáticas (quejas) que pueden no ser reconocidas por el empleador, el ingeniero en prevención, el inspector, o por el organismo administrador (aseguradora). Sin dudas, identificar los riesgos es un primer paso, enriqueciendo estos riesgos con las representaciones sociales (Brunet et al., 2005) 
de los participantes. El dirigente sindical logra enriquecer las problemáticas con los conocimientos aportados por los ergónomos y con sus pares dirigentes, mejorando los fundamentos que presentará al empleador y a los organismos en prevención de SST, avanzando en las representaciones colectivas y sociales (Brunet et al., 2005). Este proceso de aprendizaje es reforzado con el repertorio de estrategias (conocimiento del oficio) que tienen los distintos participantes de la formación.

En otro sentido, dentro de los aspectos de esta modalidad de formación, cabe destacar que cada vez es más difícil realizar este tipo de formación-acción para dirigentes sindicales, tomando en cuenta todos los sectores productivos y de servicios. La acción sindical goza de la eficacia que puede tener en mejorar las condiciones de los colectivos de trabajo (Boix \& Vogel, 2013), pero, en algunas empresas con prestaciones de servicios empresariales a distancia, han generado sistemas de trabajo atípicos, con relaciones más laxas a nivel interpersonal, donde la organización de trabajadores, la identificación con las personas que los representen, es más distendida y a juicio de esos trabajadores pueden ser innecesaria la acción sindical. Sin embargo, dentro de un marco histórico de la evolución de las condiciones laborales, significa un retroceso en temas de SST. Estos fenómenos que han obligado a las empresas a delegar actividades accesorias, tradicionalmente atendidas internamente, a terceros (empresas o particulares), a fin de reducir costos y concentrar recursos estratégicos en el núcleo de negocios, está marcado por los procesos implantados por el modelo macroeconómico que encontramos en nuestros países.

Es claro que una limitante de esta publicación es que se considera para el análisis sólo 6 rubros, no obstante, su elección obedece a la decisión dar mayor visibilidad a otros sectores que frecuentemente no son atendidos en materia de ergonomía y SST (Astudillo \& Ibarra, 2014). Es ampliamente conocido el contexto en el rubro de la gran minería (Manky, 2018), por ejemplo, donde los trabajadores son reconocidos por recibir importantes bonos como incentivos para la producción, lo cual ha logrado permear no sólo a las actividades de la industria agroalimentaria, sino también en la fruticultura, la pesca, en los servicios y en el transporte.

\section{Conclusiones}

61 La evidencia presentada por medio de las verbalizaciones de los participantes de las formaciones en ergonomía y género han mostrado la existencia de un modelo actual de relaciones laborales que estimula la competitividad entre las personas, basándose en la reducción de costos laborales y el aumento de beneficios a corto plazo para la empresa, que está en oposición a los objetivos que busca la SST y la ergonomía. No obstante, con la creciente competencia a nivel nacional e internacional de los mercados, es probable que el trabajo con incentivos continúe y que aumenten las escalas de bonos por producción.

62 Es necesario proponer más formaciones para los dirigentes sindicales e inspectores de SST, con una perspectiva sistémica sobre el Trabajo y la Salud, promoviendo a la participación y el diálogo entre los interesados en el mejoramiento de las condiciones de trabajo, reflejando la realidad laboral y la identificación de soluciones apropiadas para el contexto.

63 Cabe señalar que la formación se centra en los aspectos de la movilización de los conocimientos técnicos entregados en los fundamentos científicos; las estrategias 
implementadas por los dirigentes sindicales en el curso del petitorio, en cuanto a la participación de los trabajadores es un medio clave para promover un nivel más justo e igualitario en la toma de decisiones dentro de las empresas en SST, teniendo en cuenta que existe un contexto laboral determinado por las relaciones del ambiente social del trabajo, las cuales se caracterizan por ser asimétricas y de desigualdad.

Con la formación en ergonomía y género, ambos grupos de participantes pudieron poner en práctica cómo se construyen la dimensión de género en SST, perspectiva que comprende las características culturales que definen el comportamiento en el trabajo de hombres y mujeres y las relaciones entre ellos, pudieron avanzar en sus representaciones, en cómo se reparten las tareas y responsabilidades particulares en cada lugar de trabajo y la influencia que tienen los prejuicios de género al momento de organizar el trabajo.

Para los participantes de esta formación, implicó una instancia constructiva de análisis crítico de la actividad de trabajo, abriendo un espacio para la discusión y la retroalimentación entre los actores sociales de la Salud Ocupacional. Este tipo de formación-acción en ergonomía y género, debería replicarse para acceder a otros grupos de trabajadores y profundizar el proceso de análisis, ya que el actor sindical sigue siendo muy débil y los inspectores y necesitan ampliar las formas de evaluar el trabajo. Por lo tanto, el incrementar y mejorar sus conocimientos puede colaborar en beneficiar a los trabajadores y trabajadoras directa o indirectamente, generando espacios para el diálogo, cooperación de entre los participantes, intercambio de experiencias y transferencias de conocimientos. Mediante el análisis de las formaciones, hemos podido rescatar un tipo de formación que desempeña un rol fundamental en ergonomía, que es situar a la persona en actividad, desde la dimensión personal del trabajo, lo cual ha sido poco desarrollada por otras metodologías de análisis del trabajo en este ámbito en nuestro país.

\section{BIBLIOGRAFÍA}

Astudillo, P., \& Ibarra, C. (2014). La perspectiva de género, Desafíos para la Ergonomía en Chile: Una revisión Sistemática de Literatura. Ciencia \& Trabajo, 16(49), 28-37. http://dx.doi.org/10.4067/ S0718-24492014000100006

Astudillo, P., Ibarra, C., \& Medel, J. (2015). Guía de formación en ergonomía y género para dirigentes sindicales. Instituto de Salud Pública de Chile. Santiago Chile: D022-PR-500-02-001

Baril-Gingras, G., Bellemare, M., \& Brun, J. P. (2007). Conditions et processus menant à des changements à la suite d'interventions en santé et en sécurité du travail : l'exemple d'activités de formation. Perspectives interdisciplinaires sur le travail et la santé, 9-1. Consulté décembre, 11, 2018, http://journals.openedition.org/pistes/2998. DOI: 10.4000/pistes.2998

Boix, P., \& Vogel, L. (2013). Capítulo 13: Participación de los Trabajadores. In C. Ruiz-Frutos, A. García, G. Delclòs, E. Ronda, F. García (Eds.), Salud laboral: conceptos y técnicas para la prevención de riesgos laborales (pp. 151-160). Barcelona: Elsevier - Masson. ISBN: 9788445821466 
Brunet, R., Viel, M., Presselin, J., \& See, N., (2005). Le risque et la parole, construire ensemble une prévention d'acteur dans l'agriculture et l'industrie. Toulouse : Editions Octares.ISBN 2-915346-17-8

Cenich, G., \& Santos G. (2005). Propuesta de aprendizaje basado en proyectos y trabajo colaborativo: experiencia de un curso en línea. Revista Electrónica de Investigación Educativa, 7(2), 1-18. Consultado julio, 04, 2019, http://www.scielo.org.mx/scielo.php? script=sci_arttext\&pid=S1607-40412005000200004\&lng=es\&tlng=es.

Cohen, A., Colligan, M., Sinclair, R., Newman, J., \& Schuler, R. (1998). Assessing occupational safety and health training. Cincinnati, Ohio: National Institute for Occupational Safety and Health. Consultado julio, 04, 2019, https://www.cdc.gov/niosh/docs/98-145/pdfs/98-145.pdf

Dejours, C. (2009). Trabajo y Violencia. Madrid: Editorial modus laborandi.

Denis, D., Lortie, M., St-Vincent, M., Gonella, M., Plamondon, A., Delisle, A., \& Tardif, J., (2013). Participatory Training in Manual Handling - Theoretical Foundations and Proposed Approach. Report R-784. Montréal: IRSST.

Ffrench-Davis, R. (2014). Entre el neoliberalismo y el crecimiento con equidad: Cuarenta años de políticas económicas y sus lecciones para el futuro. Santiago: JC Sáez Editor.

Guérin, F., Laville, A., Daniellou, F., Duraffourg, J., \& Kerguelen, A. (2008). Comprendre le travail pour le transformer : la pratique de l'ergonomie. Toulouse : ANACT.

Herranz, J. (2015). Estudio de los fundamentos de la cooperación en la naturaleza humana desarrollados por las Ciencias Sociales. Tesis Doctoral, Instituto de Estudios de la Ciencia y la Tecnología de la Universidad de Salamanca, España. Consultado julio, 04, 2019, http://hdl.handle.net/10366/128159

Ibarra, C., \& Astudillo, P. (2014). La mobilisation dans le réseau de la santé publique du Chili : les enjeux de la prescription de la norme, de l'épidémiologie, du genre et de l'ergonomie. Actes du $45 \mathrm{e}$ congrès annuel de l'Association Canadienne D'Ergonomie (ACE), La mobilisation de l'entreprise : un enjeu méconnu de l'intervention en ergonomie et en santé au travail. Montréal, Canada, https:// www.researchgate.net/publication/

329947120_La_mobilisation_dans_le_reseau_de_la_sante_publique_du_Chili_Les_enjeux_de_la_prescription_de_la_norme_l'epidemiol Instituto Nacional de Estadísticas de Chile (2018). Banco de datos de la Encuesta Nacional de Empleo. Consultado marzo, 25, 2019, http://bancodatosene.ine.cl/

Kalleberg, A. (2011). Good Jobs, Bad Jobs: The Rise of Polarized and Precarious Employment Systems in the United States, 1970s-2000s. New York: Russell Sage Foundation.

Lacomblez, M., Ollagnier, E., \& Teiger, C. (2016). Les ergonomes peuvent-ils rester borgnes ? À propos de la relation intervention-formation-genre. Perspectives interdisciplinaires sur le travail et la santé, 18(2). Consulté décembre, 11, 2018. http://journals.openedition.org/pistes/4829. DOI: $10.4000 /$ pistes. 4829

Loriol, M., Boussard, V., \& Caroly, S. (2005). Perception et gestion du stress policier. « Situations difficiles » et prise en charge par l'institution. Les cahiers de la sécurité intérieure, 58, 213-237. Consulté décembre, 11, 2018. URL: https://halshs.archives-ouvertes.fr/halshs-00372864

Manky, O. (2018). Resource Mobilisation and Precarious Workers' Organisations: An Analysis of the Chilean Subcontracted Mineworkers' Unions. Work, Employment \& Society, 32(3), 581-598. https://doi.org/10.1177/0950017017751820

Messing, K. (2000). La Santé des travailleuses: la science est-elle aveugle? Montréal : Éditions du remue-ménage. 
Messing, K., Lefrançois, M., \& Saint-Charles, J. (2018). Observing Inequality: Can Ergonomic Observations Help Interventions Transform the Role of Gender in Work Activity? Computer Supported Cooperative Work, 1-35, First Online: 11 September. https://doi.org/10.1007/ s10606-018-9337-X

Montreuil, S., \& Lacomblez, M. (2013). La formation comme moyen d'intervention en prévention dans le domaine de la santé et de la sécurité du travail. In S. Montreuil, P. S. Fournier, \& G. BarilGingras (Dirs.), L'intervention en santé et en sécurité du travail - Pour agir en prévention dans les milieux de travail (pp. 295-316). Québec: Presses de l'Université Laval.

Premji, S., Lippel, K., \& Messing, K. (2008). « On travaille à la seconde! » Rémunération à la pièce et santé et sécurité du travail dans une perspective qui tient compte de l'ethnicité et du genre. Perspectives interdisciplinaires sur le travail et la santé, 10-1. Consulté juillet, 04, 2019, http:// journals.openedition.org/pistes/2181. DOI : 10.4000/pistes.2181

St-Vincent, M. Vézina, N., Bellemare, M, Denis, D., Ledoux, E., \& Imbeau D. (2011). L'intervention en ergonomie. Québec : Éditions MultiMondes et IRSST.

Siegrist, J. (1996). Adverse health effects of high-effort/low-reward conditions. Journal of Occupational Health Psychology, 1(1), 27-41. http://dx.doi.org/10.1037/1076-8998.1.1.27

Teiger, C., Lacomblez, M., Gaudart, C., Théry, L., Chassaing, K., \& Gâche, F. (2014). Dynamique de la compréhension et de la transformation du travail. Éléments pour une histoire de la coopération syndicats-recherche en ergonomie et psychologie du travail en France. Nouvelle revue de psychosociologie, 18(2), 195-210. https://doi.org/10.3917/nrp.018.0195

Teiger, C., \& Lacomblez, M. (Coord.) (2013). (Se) Former pour transformer le travail. Dynamiques de constructions d'une analyse critique du travail. Laval: Presses de l'Université Laval.

Theorell, T., Karasek, R. A., \& Eneroth, P. (1990). Job strain variations in relation to plasma testosterone fluctuations in working men-a longitudinal study. Journal of internal medicine, 227(1), 31-36. https://doi.org/10.1111/j.1365-2796.1990.tb00115.x

Valdés, X., Rebolledo, L., Pavez, J., \& Hernández, G. (2014). Trabajos y familias en el neoliberalismo hombres y mujeres en faenas de la uva, el salmón y el cobre. Santiago Chile : LOM Ediciones.

Vézina, N., (2001). La pratique de l'ergonomie face aux TMS: ouverture à l'interdisciplinarité. In Comptes rendus du 36e congrès SELF : Les transformations du travail, enjeux pour l'ergonomie. Montréal Canadá, 1, 44-50, https://s3.amazonaws.com/academia.edu.documents/35036059/v1-05avezina.pdf?response-content-disposition=inline\%3B\%20filename\%3DV1-05a-vezina.pdf\&X-AmzAlgorithm=AWS4-HMAC-SHA256\&X-Amz Credential=AKIAIWOWYYGZ2Y53UL3A\%2F20190704\%2Fus-east-1\%2Fs3\%2Faws4_request\&X-AmzDate $=20190704 \mathrm{~T} 233713 Z \& X-A m z-E x p i r e s=3600 \& X-A m z-$ SignedHeaders=host $\& X-A m z-$ Signature=f244c4d56dd9e7e4d8826cebfb32fe981d3ca654d5f216ddd860d37a7aeacc16.

Yvon, F. \& Durand, M. (2012). Réconcilier recherche et formation par l'analyse de l'activité. Louvain-laNeuve: De Boeck Supérieur. Doi: 10.3917/dbu.yvon.2011.01.

\section{RESÚMENES}

Chile ha sufrido una precarización del empleo desde los años 80s, exacerbando un modelo económico neoliberal y reformas laborales que han introducido nuevas formas de trabajo, denominadas atípicas, con mecanismos de remuneración basados en el trato con incentivos salariales colectivos o individuales. Los mecanismos de representación en las empresas han sido 
insuficientes para mejorar las condiciones de empleo y menos aún para reflexionar sobre cómo estas pueden influir en la salud de los trabajadores. Además, la acción sindical ha sido decreciente en este ámbito. Este contexto ha desincentivando el trabajo colectivo y la cooperación entre los trabajadores. Este artículo busca mostrar que la formación en ergonomía aporta a los dirigentes sindicales e inspectores de SST conocimientos prácticos sobre los impactos en la actividad, efectos en SST, entre los colectivos de trabajo y las consecuencias para la cooperación que tienen los cambios dentro de la organización del trabajo y los mecanismos de incentivo salarial.

O Chile sofre uma precarização do emprego desde os anos 1980, exacerbando um modelo econômico neoliberal e reformas trabalhistas que introduziram novas formas de trabalho, chamadas atípicas, com mecanismos de compensação baseados no tratamento com incentivos salariais coletivos ou individuais. Os mecanismos de representação nas empresas têm sido insuficientes para melhorar as condições de emprego e ainda menos para refletir sobre como estes podem influenciar a saúde dos trabalhadores. Além disso, a ação sindical vem diminuindo nessa área. Este contexto tem desencorajado o trabalho coletivo e a cooperação entre os trabalhadores. Este artigo tem como objetivo mostrar que a formação em ergonomia proporciona aos líderes sindicais e inspetores da SST conhecimento prático sobre os impactos na atividade, em SST, entre os coletivos de trabalho e as consequências para a cooperação que têm as mudanças na organização do trabalho e os mecanismos de incentivo salarial.

Le Chili souffre d'une précarisation de l'emploi depuis les années 80 , exacerbant un modèle économique néolibéral et des réformes du travail introduisant de nouvelles formes de travail dites atypiques, avec des mécanismes de rémunération basé sur l'accord avec des incitations salariales collectives ou individuelles. Les mécanismes de représentation dans les entreprises ont été insuffisants pour améliorer les conditions de travail et encore moins pour réfléchir à la manière dont ils peuvent influer sur la santé des travailleurs. Déplus l'action syndicale a décru dans ce domaine. Ce contexte-lâ a découragé le travail collectif et la coopération entre travailleurs. Cet article cherche montrer que la formation en ergonomie fournit aux dirigeants syndicaux et aux inspecteurs de SST des connaissances pratiques sur les impacts dans l'activité, dans la SST, entre les collectifs de travail et les conséquences pour la coopération qui ont des modifications dans l'organisation du travail et les mécanismes d'incitation salariaux.

Chile has suffered a precarization of employment since the 1980s, exacerbating a neoliberal economic model and labor reforms that have introduced new forms of work, called atypical, with remuneration mechanisms based on the deal, with collective or individual wage incentives. The mechanisms of representation in companies have been insufficient to improve employment conditions and even less to reflect on how these can influence the health of workers. In addition, union action has been decreasing in this area. This context has discouraged collective work and cooperation among workers. This article aims to show that ergonomics training contributes to union leaders and OSH inspectors a practical knowledge about the impacts on the activity and OSH, among the collectives of work and the consequences for cooperation that have changes in the organization of work and incentive mechanisms salary. 
ÍNDICE

Palabras claves: incentivos salariales, colectivos de trabajo, cooperación, formación-acción, ergonomía

Mots-clés: incitations salariales, collectifs de travail, coopération, formation-action, ergonomie Palavras-chave: incentivos salariais, coletivos de trabalho, cooperação, formação-ação, ergonomia

Keywords: wage incentives, collectives of work, cooperation, training-action, ergonomics

\section{AUTORES}

\section{PAMELA ASTUDILLO}

Departamento de Kinesiología de la Facultad de Ciencias de la Salud, Universidad de Atacama; Copayapu 2872, Copiapó-Chile, código postal: 1533722 pamela.astudillo@uda.cl

\section{CARLOS IBARRA}

Departamento de Kinesiología de la Facultad de Ciencias de la Salud, Universidad de Atacama; Copayapu 2872, Copiapó-Chile, código postal: 1533722

carlos.ibarra@uda.cl 